\title{
Impact of Electrodes on Recombination in Bulk Heterojunction Organic Solar Cells
}

\author{
Azadeh Rahimi Chatri, ${ }^{\circledR}$ Solmaz Torabi, ${ }^{\circledR}$ Vincent M. Le Corre, and L. Jan Anton Koster*® \\ Zernike Institute for Advanced Materials, University of Groningen, Nijenborgh 4, 9747 AG Groningen, The Netherlands
}

Supporting Information

ABSTRACT: In recent years, the efficiency of organic solar cells (OSCs) has increased to more than 13\%, although different barriers are on the way for reaching higher efficiencies. One crucial barrier is the recombination of charge carriers, which can either occur as the bulk recombination of photogenerated charges or the recombination of photogenerated charges and electrodic induced charges (EICs). This work studies the impact of EICs on the recombination lifetime in OSCs. To this end, the net recombination lifetime of photogenerated charge carriers in the presence of EICs is measured by means of conventional and newly developed transient photovoltage techniques. Moreover, a new approach has been introduced to exclusively measure the bulk recombination lifetime, i.e., in the absence of EICs; this approach was conducted by depositing transparent insulating layers on both sides of the OSC active layer. An examination of these approaches on OSCs with different active layer materials, thicknesses, and varying light intensities determined that the EICs can only reduce the recombination lifetime of the photogenerated charges in OSCs with very weak recombination strength. This work supports that for OSCs with highly reduced recombination strength, eliminating the recombination of photogenerated charges and EICs is critical for achieving better performance. Therefore, the use of a proper blocking layer suppresses EIC recombination in systems with very weak recombination.

KEYWORDS: organic solar cells, recombination lifetime, induced charges, blocking layers, bulk heterojunction

\section{INTRODUCTION}

The best organic photovoltaic devices have reached power conversion efficiencies of more than $13 \% .^{1}$ The voltage loss in solar cells is attributed to the recombination of photoinduced charges in the absorber bulk, which reaches its maximum at open circuit. ${ }^{2}$ Due to the smaller field in the device near open circuit, the extraction of charges is much more difficult than at short circuit. This effect results in much higher charge carrier densities and a large charge carrier loss due to recombination. Therefore, reducing recombination losses is of great importance to achieve higher-efficiency organic solar cells. ${ }^{3-5}$ The two pathways of recombination are the bulk recombination of photogenerated charges and the recombination of photogenerated charges with electrodic induced charges (EIC recombination). Bulk recombination of photogenerated opposite charge carriers occurs in the bulk of the active layer. EIC recombination may significantly impact device performance and reduce the net recombination lifetime. To approach this problem, it is necessary to separate and measure the two pathways of recombination.

The bimolecular recombination rate $(R)$ in organic solar cells can be described by the equation ${ }^{3}$

$$
R=\gamma\left(n p-n_{\mathrm{i}}^{2}\right)
$$

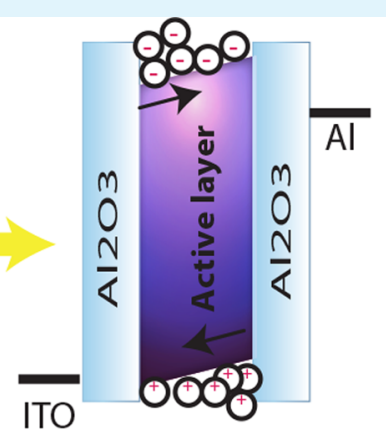

ITO

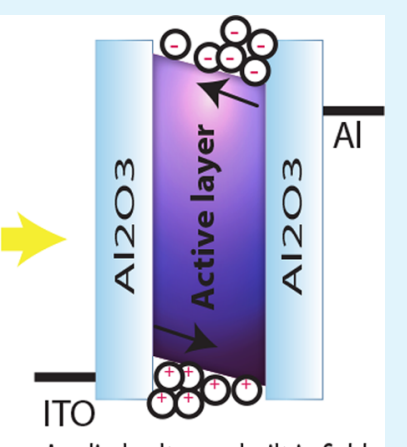

Applied voltage $<$ built-in field the intrinsic charge carrier density $n_{\text {iv }}$ and the bimolecular recombination coefficient $\gamma$, which is given by the modified Langevin equation $^{6}$

$$
\gamma=\gamma_{\mathrm{pre}} \frac{q}{\varepsilon}\left(\mu_{n}+\mu_{p}\right)
$$

where $\gamma_{\text {pre }}$ is the Langevin prefactor, $q$ is the elementary charge, $\varepsilon$ is the dielectric constant, and $\mu_{n}$ and $\mu_{p}$ are the mobilities of electrons and holes, respectively. Most polymer/fullerene bulk heterojunction $(\mathrm{BHJ})$ systems studied to date have a $\gamma_{\text {pre }}$ between 0.01 and $1 .^{7-13}$ There are some reports for P3HT:PCBM solar cells showing a $\gamma_{\text {pre }}$ as low as $10^{-314,15}$ and for polymer/nonfullerene $\mathrm{BHJ}$ solar cells as low as $10^{-4} \cdot{ }^{16}$

Several methods have been used to determine the charge carrier mean lifetime and the recombination rate coefficient $\gamma$ in solar cells by means of time-resolved techniques such as transient photovoltage (TPV), ${ }^{17-22}$ transient absorption spectroscopy, ${ }^{23}$ transient photocurrent, ${ }^{18}$ charge extraction, ${ }^{17-19,21,22}$ impedance spectroscopy, ${ }^{20}$ and time delayed

Received: December 18, 2017

Accepted: March 16, 2018

Published: March 16, 2018 
collection field methods. ${ }^{24-26}$ An analytical model to analyze recombination losses as a function of light intensity has also been introduced. ${ }^{27}$ Furthermore, the role of recombination at the interfaces and contacts has been widely studied either theoretically or experimentally by using different electrode structures, film nanomorphology, etc. ${ }^{17,22,25,28-34}$ Many BHJ solar cells have improved from the use of hole/electron blocking layers. A wide range of explanations on their effect have been proposed, including preventing recombination of minority carriers at the interface between active layer and electrode, enhancement in charge carrier mobility, increasing the built-in voltage and therefore a better charge collection efficiency, greater stability, and enhanced blocking phenomena due to formation of surface dipoles at anode (cathode) interfacial layer and acceptor (donor), etc. ${ }^{35-40}$ On the other hand, it has been reported that the presence of charge-injecting contacts reduces the forward photocurrent due to the EIC recombinaton. ${ }^{32,41}$ However, a conclusive study on the role of blocking layers in reducing EIC recombination is lacking in the literature.

To this end, we need to separate the contributions due to bulk and EIC recombination. To the best of our knowledge, no straightforward experimental way has been utilized to differentiate between the bulk recombination lifetime and net recombination lifetime (bulk plus EIC recombination). Therefore, the measured bimolecular recombination lifetimes include both bulk and EIC recombination, making it impossible to exclusively measure the bulk recombination lifetime of the photogenerated charges.

Our approach can be illustrated by considering that the free charge carrier densities $(n, p)$ can be written as a sum of the density of photogenerated charges plus the density of darkinduced charges

$$
n=n_{\mathrm{ph}}+n_{\mathrm{d}}
$$

for electrons, and

$$
p=p_{\mathrm{ph}}+p_{\mathrm{d}}
$$

for holes with the photogenerated electrons $n_{\mathrm{ph}}$, photogenerated holes $p_{\mathrm{ph}}$, dark-induced electrons $n_{\mathrm{d}}$, and darkinduced holes $p_{\mathrm{d}}$. Therefore, using eq 1 , the contribution of electrodes to the measured recombination rate is as follows

$$
R=\gamma\left(n_{\mathrm{ph}} p_{\mathrm{ph}}+\left(n_{\mathrm{ph}} p_{\mathrm{d}}+p_{\mathrm{ph}} n_{\mathrm{d}}+n_{\mathrm{d}} p_{\mathrm{d}}-n_{\mathrm{i}}^{2}\right)\right)
$$

This expression for the recombination rate makes clear that induced charges contribute to the recombination of photogenerated ones. In the theoretical determination of the bimolecular recombination coefficient, the omission of such induced charges could result in overestimation of $\gamma .{ }^{25}$ Although its derivation assumes bimolecular recombination to be the main process, eq 5 is more general and would also apply, mutatis mutandis, when trap-assisted recombination dominates. Herein, we physically isolate the active layer from the electrodes by depositing an aluminum oxide $\left(\mathrm{Al}_{2} \mathrm{O}_{3}\right)$ transparent insulating layer on both sides. Therefore, $n_{\mathrm{d}}$ and $p_{\mathrm{d}}$ are not present in the active layer and make no contribution to the measured recombination lifetime. Bulk recombination lifetime is measured from the exponential decay of the displacement current density after discharging the photocapacitor by a small reduction in light intensity. The value of $\gamma$ is then measured using a well-defined formula, the derivation of which will be explained in Section 2.1. We determined a good match between the measured values of $\gamma_{\text {pre }}$ for blends of P3HT:[60]PCBM, PDPP5T:[60]PCBM, and PTB7:[70]PCBM with the previously reported values of $\gamma_{\text {pre }}$ for these materials. ${ }^{42}$

We further employed conventional and a newly developed transient photovoltage technique, to measure the net recombination lifetime of the photogenerated charge carriers in the presence of induced charges. A detailed explanation of the basic principles and working mechanisms of our method will be given in Section 2.

EIC recombination results in a shorter net recombination lifetime than a bulk recombination lifetime. Our simulation studies show that EICs reduce the recombination lifetime in solar cells with a $\gamma_{\text {pre }}$ of less than $10^{-3}$. A shorter net recombination lifetime in an active layer with a highly reduced $\gamma_{\text {pre }}$ might be related to the accumulation of photogenerated minority charges near the electrodes, which potentiates the injection of more opposite charges under illumination, leading to increased recombination near the electrodes. Finally, we conclude that the positive role of blocking layers in reducing EIC recombination is limited to the systems with extremely low $\gamma_{\text {pre }}$

\section{THEORETICAL BACKGROUND}

2.1. Measurement of the Bulk Recombination Lifetime and Recombination Coefficient. To measure bulk recombination lifetime of photogenerated charges, a new contactless technique was developed, in which the active layer is sandwiched between two insulating layers. As shown in Figure 1, the blocked devices were made with device configuration of indium tin oxide (ITO) $/ \mathrm{Al}_{2} \mathrm{O}_{3}(40 \mathrm{~nm}) /$ active layer $/ \mathrm{Al}_{2} \mathrm{O}_{3}(40 \mathrm{~nm}) / \mathrm{Al}(100 \mathrm{~nm})$.

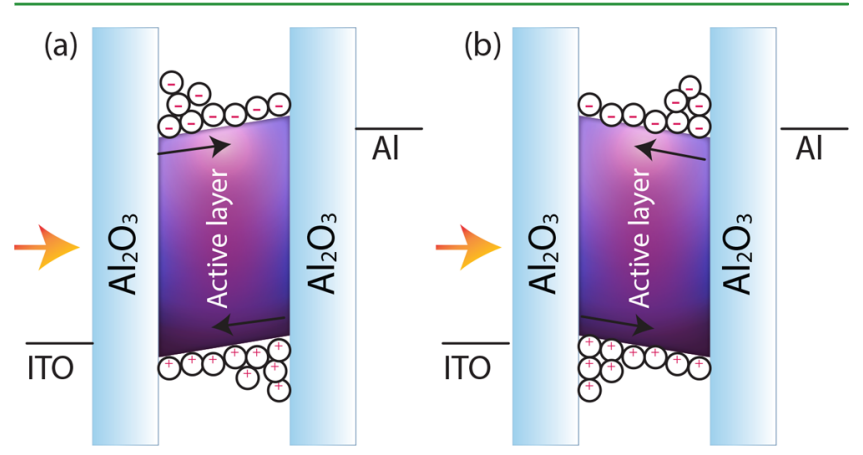

Figure 1. Schematic illustration of the sign of the displacement current after reducing the light intensity. (a) $V_{\text {app }}$ larger than $V_{\mathrm{bi}}$ results in a negative displacement current and (b) $V_{\mathrm{app}}$ smaller than $V_{\mathrm{bi}}$ results in a positive displacement current.

In the transport and recombination via the displacement current (TRDC) experiment, we aimed to measure the recombination lifetime via a small perturbation in the intensity of incident light. We started by placing the photocapacitor under steady-state illumination. At time 0 , the light intensity was slightly reduced using a wave form of a step function, and the resulting displacement current was measured. As the light intensity was abruptly reduced, the excess charge carriers recombine until a steady state is reached at this lower light intensity (Figure 1).

This recombination means that the polarization of the capacitor is reduced, and hence a displacement current flows. Figure S1 illustrates the TRDC setup and steps involved during the experiment. A simple analytical expression for the 
displacement current can be obtained as follows. At time 0 , we have a steady-state situation with the generation rate $G+\Delta G$. As this is steady state, generation and recombination cancel, and we have $R+\Delta R=G+\Delta G$, where $\Delta R$ is the recombination of excess carriers. As the light intensity is reduced to $G$ and after sufficient time, we again have $G=R=\gamma n p$, where $\gamma$ is the bimolecular recombination rate constant, and $n$ and $p$ are the electron and hole densities, respectively, of photogenerated charges at steady state. At time 0 , the densities of electrons and holes are slightly larger (due to more light) and we have

$$
R+\Delta R=\gamma(n+\Delta n)(p+\Delta p) \approx \gamma(n p+p \Delta n+n \Delta p)
$$

As the second-order term $(\Delta n \Delta p)$ is smaller than the other terms, it can be neglected in the expression of eq 6 . Thus, using eq 6 , we can write the decay of the charge carrier densities as

$$
\begin{gathered}
\frac{\mathrm{d}(n+\Delta n)}{\mathrm{d} t}=\frac{\mathrm{d} \Delta n}{\mathrm{~d} t}=G-(R+\Delta R) \\
=G-\gamma(n p+p \Delta n+n \Delta p)
\end{gathered}
$$

In addition, as the electrons and holes are generated (and recombine) as a pair, the electron and hole concentrations are equal, such as $n=p$ and $\Delta n=\Delta p$. During the experiment, $n$ and $p$ are constant due to the illumination $G$. By writing $G=R$, the electron and hole densities can be written as $n=p=\sqrt{G / \gamma}$. Therefore, eq 7 becomes

$$
\frac{\mathrm{d} \Delta n}{\mathrm{~d} t}=-\tau^{-1} \Delta n
$$

where the lifetime can be expressed as a function of $G$ and $\gamma$ as follows

$$
\tau^{-1}=2 \sqrt{G \gamma}
$$

As a result, the current can be written in the same way, as it is proportional to the decay of the charge carriers

$$
J \propto \exp (-2 \sqrt{G \gamma} t)
$$

Therefore, we can fit a simple monoexponential function to the decay of the current to measure the recombination rate coefficient $\gamma$.

Note that the lifetime in eq 9 does not depend on the applied voltage. A simulated plot of displacement current density under varying applied voltages is shown in Figure 2.

Figure 1 shows the reason that the sign of displacement current density switches at different voltages. The built-in voltage $\left(V_{\mathrm{bi}}\right)$ pushes photogenerated electrons to the cathode side and holes to the anode side. When the applied voltage $\left(V_{\text {app }}\right)$ is larger than $V_{\mathrm{bi}}$ in the device, the excess photogenerated electrons (holes) are pushed toward the interface between the active layer and the $\mathrm{Al}_{2} \mathrm{O}_{3}$ blocking layer at the anode (cathode) side. Therefore, the displacement current density is negative (Figure 1a). In contrast, when $V_{\text {app }}$ is smaller than $V_{\mathrm{bi}}$ the displacement current density switches to positive, as the charges recombine in the opposite direction by applying the perturbation of light intensity (Figure $1 \mathrm{~b}$ ). The larger the difference is between $V_{\mathrm{app}}$ and $V_{\mathrm{bi}}$ the more charging of the capacitor, which causes a larger displacement current flow. At voltages very close to $V_{\mathrm{bi}}$, the fields more strongly compensate each other, and therefore a very low amplitude displacement current density is observed. The recombination lifetime $\left(\tau_{\mathrm{TRDC}}\right)$ is measured by fitting the monoexponential decay of

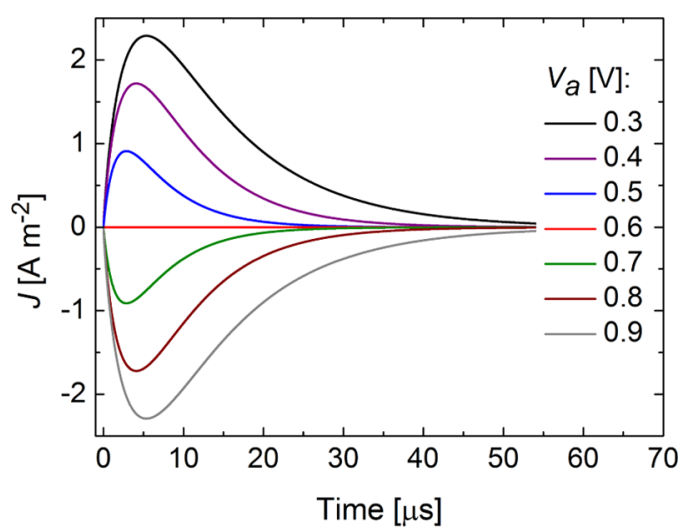

Figure 2. Numerical drift-diffusion simulation graph of the displacement current density versus time at various applied voltages, showing an exponential decrease after the rise at early times by the reduction of light intensity.

the displacement current density at a range of applied voltages of around $V_{\mathrm{bi}}(-2$ to $2 \mathrm{~V})$. The use of eq 9 allows the recombination coefficient $\gamma$ to be calculated.

2.2. Transient Photovoltage Measurements. To investigate the effect of induced charges on the recombination lifetime in studied solar cells, two different techniques were used. The transient photovoltage decay lifetime of the solar cells (TPV) was measured under a small perturbation of lightemitting diode (LED) illumination intensity. The high input impedance of the oscilloscope $(1 \mathrm{M} \Omega)$ was used to provide an open circuit condition under LED illumination.

In the modified version of TPV (TPV2), first, the charge decay was measured by monitoring the displacement current density transients over a capacitor $(C)$ placed in series with the solar cell under a constant applied bias $(V)$ and a pulsed light LED. Note that the illumination condition is the same as in the TPV and TRDC methods. Then, the capacitor was varied. The intercept of $\tau$ versus $C$ gives the net recombination lifetime of the charge carriers in the solar cell in the presence of EICs. Schematic representations of TPV and TPV2 setup are shown in Figure S2. Next, we define the requirements of TPV2 by providing a detailed explanation of the working mechanism of the method.

At time 0 , when the device is under steady state condition, the light intensity is reduced. Therefore, the application of a small perturbation of LED light intensity causes a decrease in $V_{\mathrm{OC}}$. The potential across the series capacitor $\left(V_{\mathrm{C}}\right)$ equals

$$
V_{\mathrm{C}}=V-V_{\mathrm{SC}}=V-V_{\mathrm{OC}}
$$

where $V_{S C}$ is the potential across the solar cell. The applied voltage on the whole device $(V)$ is kept constant. Therefore, the potential drop across the series capacitor $\left(\Delta V_{\mathrm{C}}\right)$ always equals $\Delta V_{\mathrm{OC}}$

$$
\Delta V_{\mathrm{C}}=\Delta V-\Delta V_{\mathrm{OC}}=-\Delta V_{\mathrm{OC}}
$$

Because the series capacitance stays constant, the potential drop due to the reduced light intensity causes charging of the capacitor, which linearly increases with $\Delta V_{\mathrm{OC}}$

$$
\Delta Q_{\mathrm{C}}=C \Delta V_{\mathrm{C}}=-C \Delta V_{\mathrm{OC}}
$$

On the basis of eq 13 , the slope of $\Delta Q_{\mathrm{C}}$ versus $C$ equals $\Delta V_{\mathrm{OC}}$ (see Figure S3, Supporting Information), which is in good agreement with the direct measurement of $V_{\mathrm{OC}}$ of the solar cells at higher and lower intensities (see Table S1, Supporting 

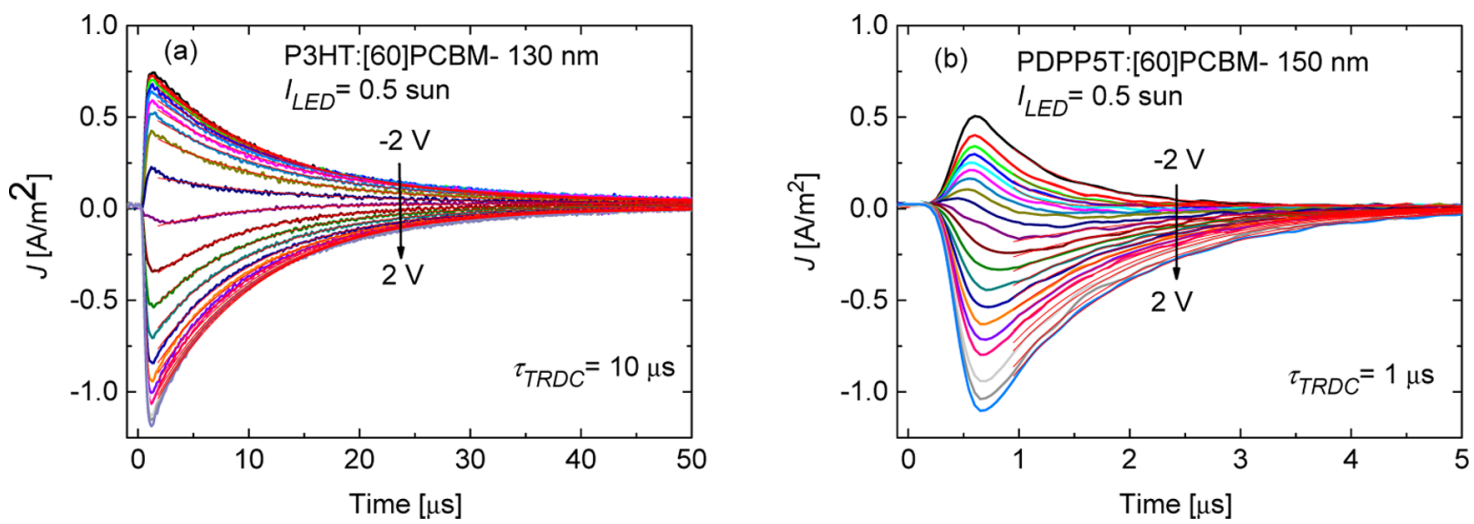

Figure 3. Comparison between TRDC lifetimes, acquired for (a) blocked P3HT:[60]PCBM and (b) blocked PDPP5T:[60]PCBM devices under $\sim 0.5$ sun LED light intensity. The mean lifetime is derived from the mean value of the time constants from fitted exponential decays of the displacement current densities versus time at various voltages (Figure S9, Supporting Information).

Table 1. TRDC Parameters under Various Generation Rates G, Measured for Blocked Devices of P3HT: 60$]$ PCBM and PDPP5T:[60]PCBM

\begin{tabular}{cccccc} 
& $d(\mathrm{~nm})$ & $G\left(\mathrm{~m}^{-3} \mathrm{~s}^{-1}\right)$ & $\tau_{\text {TRDC }}(\mu \mathrm{s})$ & $\gamma\left(\mathrm{m}^{3} \mathrm{~s}^{-1}\right)$ & $\gamma_{\text {pre }}$ \\
P3HT:[60]PCBM & 130 & $1.50 \times 10^{27}$ & $10 \pm 2.3$ & $1.6 \times 10^{-18}$ & $1.21 \times 10^{-3}$ \\
& & $6.00 \times 10^{26}$ & $18 \pm 3.8$ & $1.4 \times 10^{-18}$ & $1.06 \times 10^{-3}$ \\
& & $1.50 \times 10^{26}$ & $38 \pm 8.9$ & $1.2 \times 10^{-18}$ & $9.00 \times 10^{-4}$ \\
P3HT:[60]PCBM & 260 & $1.07 \times 10^{27}$ & $15 \pm 3.4$ & $1.1 \times 10^{-18}$ & $8.30 \times 10^{-4}$ \\
& & $4.30 \times 10^{26}$ & $25 \pm 5.5$ & $9.7 \times 10^{-19}$ & $7.30 \times 10^{-4}$ \\
PDPP5T:[60]PCBM & & $1.10 \times 10^{26}$ & $102 \pm 26$ & $2.3 \times 10^{-19}$ & $1.70 \times 10^{-4}$ \\
& \multirow{2}{*}{150} & $3.38 \times 10^{27}$ & $1 \pm 0.2$ & $2.3 \times 10^{-16}$ & $6.31 \times 10^{-2}$ \\
& & $1.35 \times 10^{27}$ & $2 \pm 0.4$ & $1.8 \times 10^{-16}$ & $5.06 \times 10^{-2}$ \\
& & $3.30 \times 10^{26}$ & $7 \pm 2.0$ & $6.7 \times 10^{-17}$ & $1.85 \times 10^{-2}$
\end{tabular}

Information). Corresponding $J-V$ curves are also shown in the Supporting Information (see Figure S4).

2.3. Light Intensity-Dependent Measurements. Measuring the light intensity-dependent bulk-only (using TRDC) and net recombination lifetimes (using TPV and TPV2) enables a better understanding of the influence of recombination with induced charges on the recombination lifetime. $V_{\mathrm{OC}}$ is related to light intensity by the following equation ${ }^{38,39}$

$$
V_{\mathrm{OC}}=\frac{n k T}{q}\left(\ln \left(\frac{J_{\mathrm{SC}}}{J_{\mathrm{s}}}\right)+1\right)
$$

where $J_{\mathrm{s}}$ is the dark saturation current density, $J_{\mathrm{SC}}$ is the lightgenerated current density, $n$ is the ideality factor, and $k$ is the Boltzmann's constant. For the condition, in which the light intensity decreases from high to low, we can calculate $\Delta V_{\mathrm{OC}}$ using the following equation

$$
\Delta V_{\mathrm{OC}}=\frac{n k T}{q} \ln \left(\frac{G+\Delta G}{G}\right)
$$

where $G+\Delta G$ and $G$ correspond to generation rates at higher and lower illumination intensities. Therefore, using eq 13, we have

$$
\Delta Q_{\mathrm{C}}=-C \frac{n k T}{q} \ln \left(\frac{G+\Delta G}{G}\right)
$$

According to eq 16, varying $G$ while keeping the ratio $(G+$ $\Delta G) / G$ constant allows $\Delta Q_{C}$ to remain constant. This means by integrating the displacement current over the light perturbation time $(5 \mathrm{~ms})$, the same number of photogenerated charge carriers recombine (see Figure S5, Supporting Information). Here, $G$ was varied by using optical filters. $G$ is calculated by $G=\frac{J_{\mathrm{SC}}}{q \times d}$, where $J_{\mathrm{SC}}$ is the short circuit current density of the solar cell under steady-state LED illumination at a lower intensity and $d$ is the thickness of the active layer. Reducing $G$ causes an increased bulk recombination lifetime of excess photogenerated charges measured by TRDC, whereas the rate of recombination with induced charges does not depend on the light intensity.

\section{RESULTS AND DISCUSSION}

3.1. Bulk Recombination Lifetime and the Recombination Coefficient. $J-V$ curves of blocked devices show no leakage current (see Figure S6). Figure 3 shows TRDC data on blocked P3HT:[60]PCBM and PDPP5T:[60]PCBM devices under a light intensity of $\sim 0.5$ sun. LED light intenstiy was estimated by the ratio of the value of $J_{\mathrm{SC}}$ of the device under LED illumination to the value of $J_{S C}$ at 1 sun. Complementary TRDC data as a function of LED light intensity together with all TRDC data for P3HT:[60]PCBM $(260 \mathrm{~nm})$ are provided in the Supporting Information (Figures S7 and S8). The applied voltage on the device varied between -2 and $2 \mathrm{~V}$, with steps of $0.2 \mathrm{~V}$. For each light intensity, the TRDC mean lifetime is derived by averaging the measured lifetimes from monoexponential decay fits of the displacement current densities versus time within the voltage range of $\left|V_{\text {app }}\right|>1 \mathrm{~V}$, which are due to obtaining of small amplitude signals close to 0 for the conditions where the field in the device is almost compensated by the built-in field (see Figure 2). At very low light intensities of approximately 0.05 sun, the average $\tau_{\mathrm{TRDC}}$ values have larger 

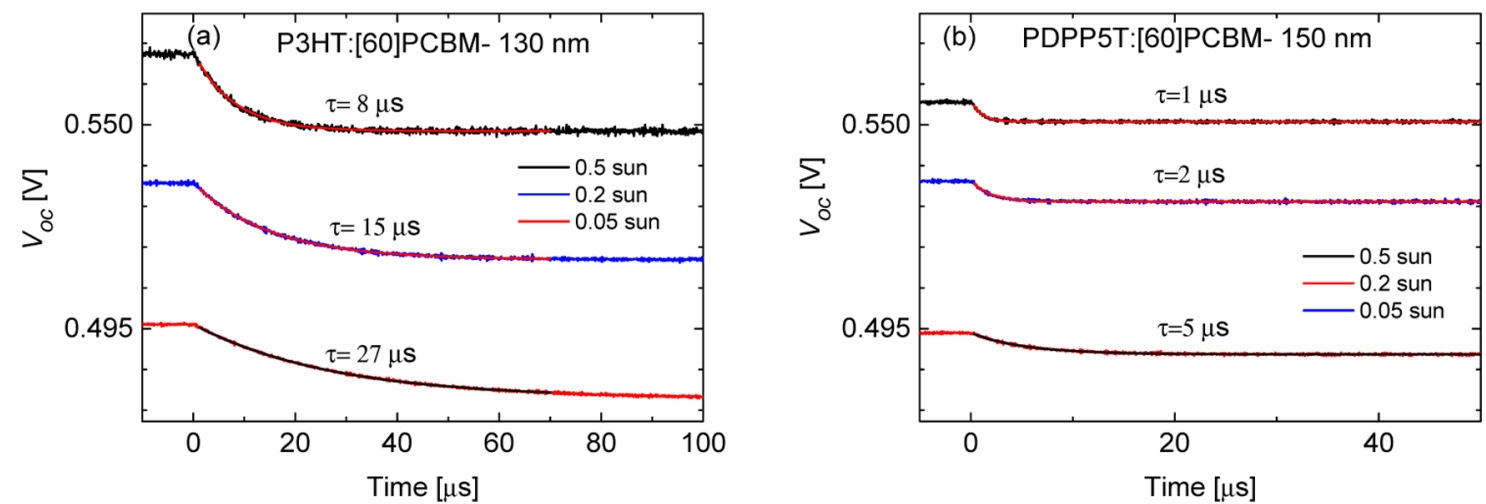

Figure 4. TPV transients as a function of light intensity for (a) P3HT:[60]PCBM (130 nm) and (b) PDPP5T:[60]PCBM (150 nm) solar cells. The lifetime is measured by fitting the exponential $V_{\mathrm{OC}}$ decay after applying a small perturbation of light by reducing the LED light intensity. TPV transients obtained for P3HT:[60]PCBM $(260 \mathrm{~nm})$ are shown in Figure S11 in the Supporting Information.
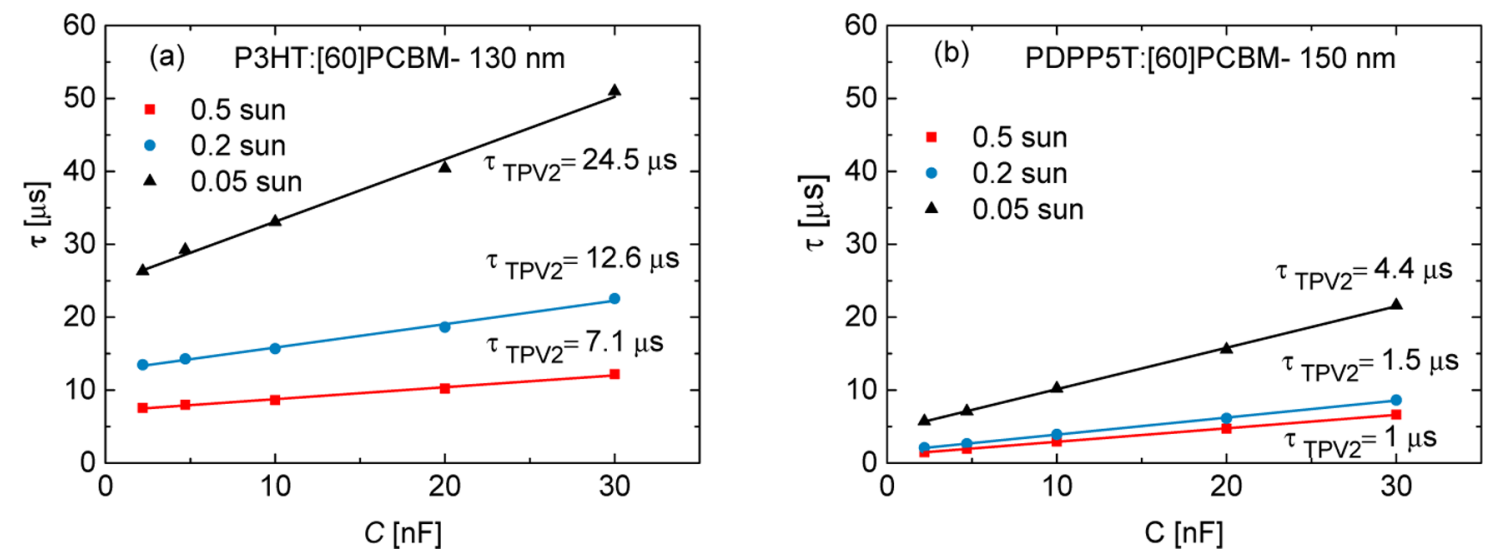

Figure 5. Recombination lifetimes using TPV2 measured at a $C=0$ condition $\left(\tau_{\mathrm{TPV} 2}\right)$ under light intensities of 0.05 sun (the black symbols), 0.2 sun (the blue symbols), and 0.5 sun (the red symbols) for (a) P3HT:[60]PCBM (130 nm) and (b) PDPP5T:[60]PCBM devices. The corresponding plot for P3HT:[60]PCBM $(260 \mathrm{~nm})$ is shown in Figure S12 in the Supporting Information.
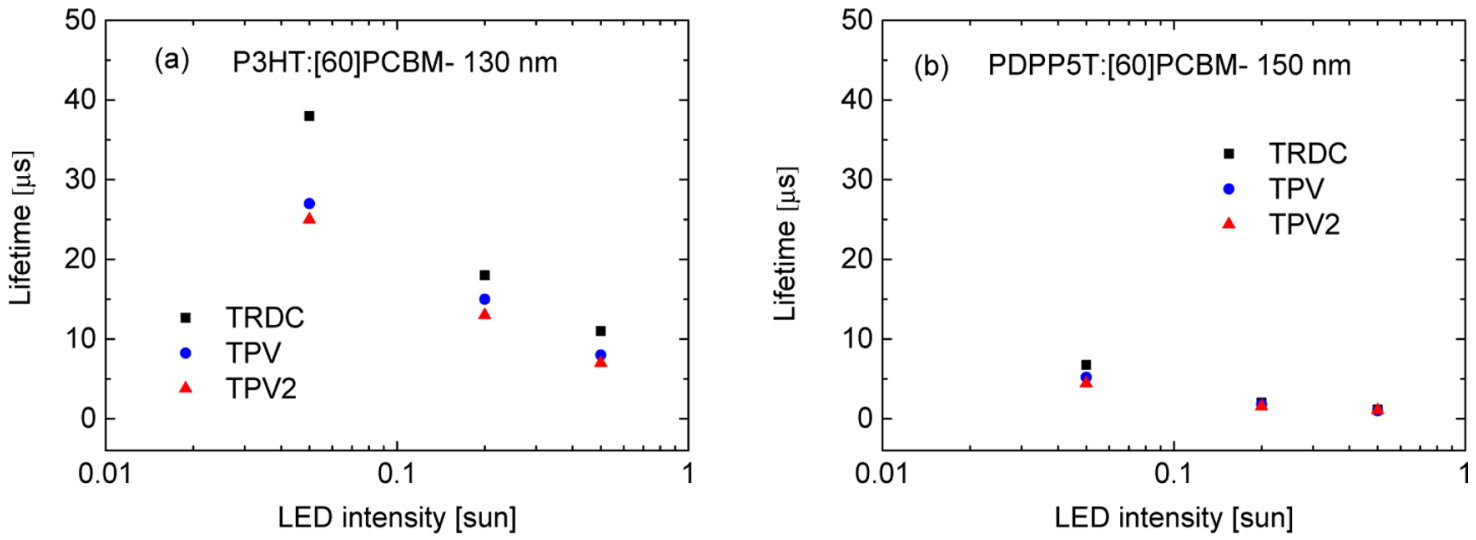

Figure 6. Comparison of the measured recombination lifetimes using different methods versus the LED light intensity for (a) P3HT:[60]PCBM and (b) PDPP5T:[60]PCBM devices. Light intensity-dependent lifetimes using the three methods for P3HT:[60]PCBM (260 nm) are shown in Figure S14 in the Supporting Information.

standard errors, as shown in Table 1, which might be the reason for variations among the values of $\gamma_{\text {pre }}$ of a single device under different light intensities.

Table 1 shows the TRDC parameters of blocked devices under the various generation rates G. According to eq 9, the lifetime is inversely proportional to the square root of the generation rate, whereas the recombination coefficient $\gamma$ is constant. Confirming our theory, the lifetime increases with decreasing intensity. Having the experimental $\tau_{\text {TRDC }}$ values, we derive the values of $\gamma$ by using eq 9, which are in good agreement with the values of $\gamma$ for these materials reported in the literature. ${ }^{42}$ Taking the charge carrier mobilities of the studied systems from the literature, ${ }^{42}$ we calculate the Langevin recombination prefactors $\left(\gamma_{\text {pre }}\right)$. At very low light intensity of approximately 0.05 sun, the average $\tau_{\text {TRDC }}$ values have larger standard errors, as shown in Table 1, which might be the reason 
for variations among the values of $\gamma_{\text {pre }}$ of a single device under different light intensities.

3.2. Transient Photovoltage Measurements. To determine the impact of EIC recombination on the net recombination lifetime in organic solar cells, two methods of TPV and TPV2 were used, as described in Section 2.2. Figures 4 and 5 show the recombination lifetimes of the studied solar cells under different light intensities using these techniques. The TPV transients (Figure 4) show an exponential $V_{\text {OC }}$ decay under an LED light perturbation. For each device, $\Delta V_{\mathrm{OC}}$ was kept constant, whereas the amplitude of small perturbation was reduced using optical filters. TPV exhibits a $\Delta V_{\text {OC }}$ decay of $\sim 0.02 \mathrm{~V}$ for P3HT:[60]PCBM and $\sim 0.005 \mathrm{~V}$ for PDPP5T: [60]PCBM. These values are consistent with the values of $\Delta V_{\mathrm{OC}}$ measured with the $J-V$ responses of the devices under steady-state conditions at higher and lower light intensities separately (see Table S1, Supporting Information). The difference among the values of $\Delta V_{\mathrm{OC}}$ for different materials is due to different ideality factors, which is confirmed by the smaller slope of $V_{\mathrm{OC}}$ versus light intensity for PDPP5T: [60]PCBM compared to P3HT:[60]PCBM (Figure S10, Supporting Information).

At lower light intensities, the lifetime increases due to the photogeneration of less excess charge in the active layer. PDPP5T:[60]PCBM exhibits a smaller recombination lifetime than P3HT:[60]PCBM under the applied light intensities. Comparing Figures 4 and 5, as expected, the recombination lifetimes measured with both TPV and TPV2 show almost the same values.

3.3. Discussion. In Figure 6, the measured mean recombination lifetimes using all the described methods under different light intensities are compared. For P3HT: [60]PCBM, the values of $\tau_{\mathrm{TRDC}}$ are slightly larger than $\tau_{\mathrm{TPV}}$ and $\tau_{\mathrm{TPV} 2}$. However, the ratio $\frac{\tau_{\mathrm{TRDC}}}{\tau_{\mathrm{TPV}}}$ is not considerably higher than the one specifically at the highest light intensity. The PDPP5T: [60] PCBM device shows equal lifetimes with the ratio $\frac{\tau_{\mathrm{TRDC}}}{\tau_{\mathrm{TPV}}}$ of 1. The light intensity-dependent measurements of the P3HT: [60] PCBM device reveal that at the lowest light intensity ( $\sim 0.05$ sun), the difference between the bulk recombination lifetime $\left(\tau_{\mathrm{TRDC}}\right)$ and the net recombination lifetime $\left(\tau_{\mathrm{TPV}}\right.$, $\left.\tau_{\mathrm{TPV} 2}\right)$ is more pronounced, because EIC recombination stays constant with light intensity, whereas the bulk recombination lifetime increases with decreasing intensity. The corresponding data of the PTB7:[70]PCBM devices are shown in the Supporting Information (Figure S13) and exhibit very similar recombination lifetimes with or without the inclusion of induced charges. Considering our measured $\gamma$ and the literature values of $\mu_{n}+\mu_{p}$ for PTB7:[70]PCBM and PDPP5T: $[60] \mathrm{PCBM},{ }^{37}$ we estimate $\gamma_{\text {pre }}$ values of $1.04 \times 10^{-2}$ and 6.3 $\times 10^{-2}$, respectively, which are 1 order of magnitude larger than $\gamma_{\text {pre }}$ of P3HT:[60]PCBM (see Table S2).

In Figure 7 , the ratio $\frac{\tau_{\mathrm{TRDC}}}{\tau_{\mathrm{TPV}}}$ is plotted versus $\gamma_{\text {pre }}$ for the devices tested in this study and are compared with the simulated data for a wide range of $\gamma_{\text {pre. }}$ The devices tested in our study have a $\gamma_{\text {pre }}$ between $10^{-3}$ and $10^{-1}$, which represents a $\gamma_{\text {pre }}$ of most of the current state of the art of solar cells. Our results show that in most of the current solar cells, the influence of EICs is negligible. However, according to the simulation, the EICs begin to play a role if bulk recombination is further suppressed.

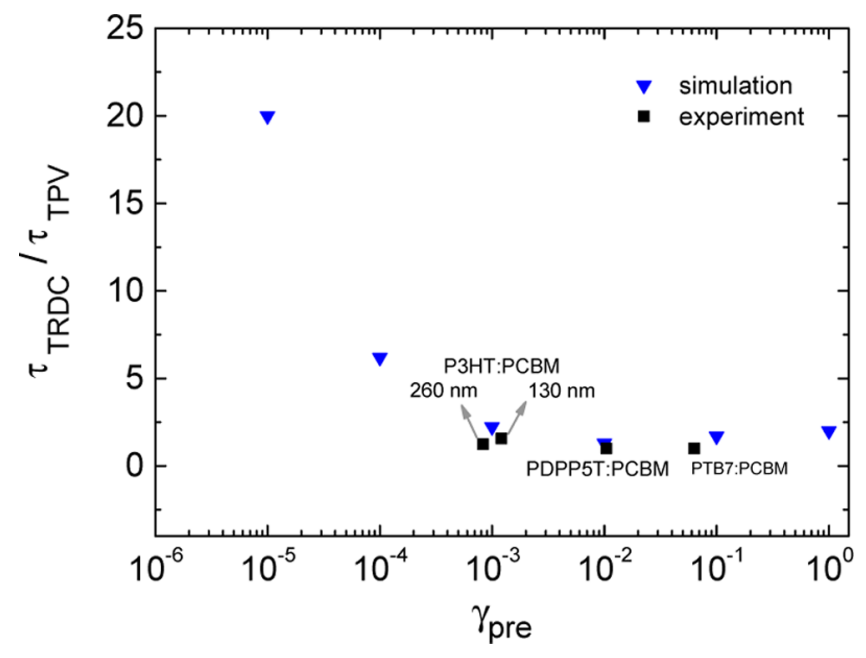

Figure 7. Ratio of bulk recombination to net recombination lifetimes versus $\gamma_{\text {pre }}$ under $\sim 0.5$ sun light intensity. Experimental points are compared with the simulations.

In solar cells with highly reduced bulk recombination strength, the shorter net recombination lifetime compared with the bulk recombination lifetime might be due to the accumulation of photogenerated minority charges near the electrodes (electrons near the anode or holes near the cathode), which enhances the injection of additional opposite charges (holes from the anode or electrons from the cathode) under illumination, leading to increased recombination near the electrodes. $^{32}$

Therefore, for most of the current state of the art organic solar cells, EICs do not considerably reduce device performance. However, note that producing organic solar cells with potentially lower $\gamma_{\text {pre }}$ values than $10^{-3}$, could result in EICs facilitating an increase in net recombination. Therefore, the use of proper blocking layers becomes very crucial to further improve device performance.

\section{CONCLUSIONS}

Although bulk recombination of photogenerated charge carriers has always been considered the main recombination pathway in organic solar cells, the recombination of charge carriers with electrodic induced charges (EIC recombination) has not been studied for many years.

In our study, a new technique was introduced to discriminate the bulk recombination of charges from EIC recombination by isolating the active layer from the electrodes using insulating layers of $\mathrm{Al}_{2} \mathrm{O}_{3}$ on both sides of the active layer. Transient TRDC decays were used to measure the bulk recombination lifetime of the photogenerated charge carriers. The net recombination lifetimes in the presence of electrodes were also measured using the TPV and newly developed TPV (TPV2) methods and compared with TRDC lifetimes for decent polymer/fullerene solar cells.

We found that in current state of the art organic solar cells, recombination with induced charges is not significant, as bulk only and net recombination lifetimes show the same values. Finally, comparing our experimental data with simulations, the results show that EIC recombination reduces the recombination lifetime for a system with a $\gamma_{\text {pre }}$ lower than $10^{-3}$. Therefore, in solar cells with highly reduced recombination strength, the use of proper blocking layers could suppress the recombination of photogenerated charges with EICs. 
The tool introduced in this paper can be used to study the contribution of recombination with induced charges near the electrodes in the net recombination lifetime of organic solar cells with very weak bimolecular recombination strength.

\section{EXPERIMENTAL PROCEDURES}

5.1. Device Preparation. In this work, two different device structures were fabricated: a bulk heterojunction $(\mathrm{BHJ})$ solar cell and a blocked device. The solar cells were fabricated using poly(3hexylthiophen-2,5-diyl) (P3HT, Rieke Metals Inc.) and PDPP5T as the donor and PCBM (purchased from Solenne) as the acceptor. Structured indium tin oxide (ITO) was used as the substrate. All substrates were cleaned with soap and water for 5 min followed by rinsing with di-ionized water and, subsequently, a 10 min treatment in an ultrasonic bath of acetone and isopropyl alcohol, separately. Finally, the substrates were spin-dried and transferred into an oven at $140{ }^{\circ} \mathrm{C}$ for $10 \mathrm{~min}$, followed by a UV-ozone treatment for $20 \mathrm{~min}$. A $50 \mathrm{~nm}$ poly(3,4-ethylenedioxythiophene):poly(styrene sulfonate) was then spin-cast on the substrate, followed by $10 \mathrm{~min}$ oven drying at $140{ }^{\circ} \mathrm{C}$ to remove the residual water.

To fabricate the P3HT:[60]PCBM solar cells $(130,260 \mathrm{~nm}$ active layer), a solution of a P3HT:[60]PCBM blend (1:1 by weight) in chloroform with a concentration of either 10 or $25 \mathrm{~g} \mathrm{~L}^{-1}$ was spincoated at 300 or $1000 \mathrm{rpm}$ for $50 \mathrm{~s}$, yielding active layers of approximately 130 and $260 \mathrm{~nm}$ thick. The active layer was then annealed at $140{ }^{\circ} \mathrm{C}$ for $5 \mathrm{~min}$. Finally, $\operatorname{LiF}(1 \mathrm{~nm})$ and $\mathrm{Al}(100 \mathrm{~nm})$ were thermally evaporated through shadow masks in a vacuum chamber at $10^{-6} \mathrm{mbar}$, defining an active area of $10 \mathrm{~mm}^{2}$.

For the PDPP5T:[60]PCBM solar cells, the blend was spin-cast from a chloroform/ortho-dichlorobenzene (5 vol \%) solution in $\mathrm{N}_{2}$ atmosphere. After drying of the polymer/fullerene film at room temperature, a cathode of $\operatorname{LiF}(1 \mathrm{~nm})$ and $\mathrm{Al}(100 \mathrm{~nm})$ was thermally evaporated.

PTB7:[70]PCBM solar cells were fabricated by spin-casting a solution of PTB7:[70]PCBM (1:1.5 by weight) in 1,2-dichlorobenzene from PTB7 $\left(16 \mathrm{~g} \mathrm{~L}^{-1}\right)$ and PCBM $\left(24 \mathrm{~g} \mathrm{~L}^{-1}\right)$. The solution was spin-coated at $600 \mathrm{rpm}$ for $120 \mathrm{~s}$, yielding an active layer of approximately $85 \mathrm{~nm}$. Finally, a cathode of $\mathrm{LiF}(1 \mathrm{~nm})$ and $\mathrm{Al}(100$ $\mathrm{nm})$ was thermally evaporated.

The blocked devices were prepared by electron beam evaporation of aluminum oxide $\left(\mathrm{Al}_{2} \mathrm{O}_{3}\right)(40 \mathrm{~nm})$ on the cleaned ITO substrate. The evaporation rate was set to $0.5 \AA \mathrm{s}^{-1}$. During the transfer to the electron beam evaporation system, the devices were exposed to air (approximately $10 \mathrm{~min}$ ). Thereafter, the active layer was spin-coated as mentioned above, followed by the evaporation of the top blocking layer of $\mathrm{Al}_{2} \mathrm{O}_{3}(40 \mathrm{~nm})$ by electron beam evaporation. Finally, aluminum $(100 \mathrm{~nm})$ was deposited as the top contact.

5.2. Measurements. Current-voltage characteristics of the solar cells were measured using a computer-controlled Keithley source meter in a $\mathrm{N}_{2}$ atmosphere. For the transient experiments, the sample was illuminated with a biased white light LED with a rise/fall time of $<200 \mathrm{~ns}$ and a frequency of $100 \mathrm{~Hz}$ with a pulse width of $5 \mathrm{~ms}$. The rise/fall time of the LED was tested using a photodiode with a $<2 \mathrm{~ns}$ rise/ fall time. Subsequent transient signals were acquired using a digital storage oscilloscope (Agilent DSO-X 3034A) with a $350 \mathrm{MHz}$ bandwidth and an input resistance of $1 \mathrm{M} \Omega$. In the TRDC experiments, a homemade circuit containing operational amplifiers and voltage switches was used. The transients were recorded by varying either the applied voltage or LED light intensity.

5.3. Simulations. TRDC and TPV simulations were obtained using a home-written transient drift-diffusion program that has already shown its ability to reproduce accurately the transient behavior of blended organic materials. ${ }^{43}$ In this model, the blend is considered to be an effective medium where the highest occupied molecular orbital (HOMO) of the effective semiconductor is taken as the HOMO value of the donor, and the lowest unoccupied molecular orbital (LUMO) of the effective semiconductor is taken as the LUMO value of the acceptor. The model describes the flow of the charge carrier considering the gradients of charge carrier concentration, diffusion, and the electrical field, drift, as driving force (for more details see refs 2 and 44). The model also takes into account charge carrier recombination using a reduced Langevin law, as it has been shown to be the dominant recombination process in state of the art organic solar cells. ${ }^{6,24,45,46}$

\section{ASSOCIATED CONTENT}

\section{S Supporting Information}

The Supporting Information is available free of charge on the ACS Publications website at DOI: 10.1021/acsami.7b19234.

$\Delta Q_{\mathrm{C}}$ plots, current-voltage parameters of the devices, complementary TRDC plots, TPV plots, and TPV2 plots (PDF)

\section{AUTHOR INFORMATION}

\section{Corresponding Author}

*E-mail: 1.j.a.koster@rug.nl.

ORCID 웅

Azadeh Rahimi Chatri: 0000-0002-2312-1425

Solmaz Torabi: 0000-0003-1612-3518

L. Jan Anton Koster: 0000-0002-6558-5295

Notes

The authors declare no competing financial interest.

\section{ACKNOWLEDGMENTS}

This work was supported by a grant from STW/NWO (VIDI 13476). The authors would like to thank Arjen Kamp and Dr. Teodor Zaharia for technical assistance. A.R.C. acknowledges Soheil Solhjoo from University of Groningen for useful discussions.

\section{REFERENCES}

(1) Zhao, W.; Li, S.; Yao, H.; Zhang, S.; Zhang, Y.; Yang, B.; Hou, J. Molecular Optimization Enables over 13\% Efficiency in Organic Solar Cells. J. Am. Chem. Soc. 2017, 139, 7148-7151.

(2) Koster, L. J. A.; Smits, E.; Mihailetchi, V.; Blom, P. W. M. Device model for the operation of polymer/fullerene bulk heterojunction solar cells. Phys. Rev. B 2005, 72, No. 085205.

(3) Proctor, C. M.; Kuik, M.; Nguyen, T. Charge carrier recombination in organic solar cells. Prog. Polym. Sci. 2013, 38, $1941-1960$

(4) Kirchartz, T.; Taretto, K.; Rau, U. Efficiency limits of organic bulk heterojunction solar cells. J. Phys. Chem. C 2009, 113, 17958-17966.

(5) Blom, P. W. M.; Mihailetchi, V. D.; Koster, L. J. A.; Markov, D. E. Device physics of polymer: fullerene bulk heterojunction solar cells. Adv. Mater. 2007, 19, 1551-1566.

(6) Langevin, P. Recombinaison et mobilites des ions dans les gaz. Ann. Chim. Phys. 1903, 28, No. 122.

(7) Pivrikas, A.; Sariciftci, N. S.; Juška, G.; Österbacka, R. A review of charge transport and recombination in polymer/fullerene organic solar cells. Prog. Photovolt: Res. Appl. 2007, 15, 677-696.

(8) Albrecht, S.; Janietz, S.; Schindler, W.; Frisch, J.; Kurpiers, J.; Kniepert, J.; Inal, S.; Pingel, P.; Fostiropoulos, K.; Koch, N.; Neher, D. Fluorinated copolymer PCPDTBT with enhanced open-circuit voltage and reduced recombination for highly efficient polymer solar cells. J. Am. Chem. Soc. 2012, 134, 14932-14944.

(9) Proctor, C. M.; Kim, C.; Neher, D.; Nguyen, T. Nongeminate Recombination and Charge Transport Limitations in Diketopyrrolopyrrole-Based Solution-Processed Small Molecule Solar Cells. Adv. Funct. Mater. 2013, 23, 3584-3594.

(10) Baumann, A.; Lorrmann, J.; Rauh, D.; Deibel, C.; Dyakonov, V. A new approach for probing the mobility and lifetime of photogenerated charge carriers in organic solar cells under real operating conditions. Adv. Mater. 2012, 24, 4381-4386. 
(11) Deibel, C.; Baumann, A.; Dyakonov, V. Polaron recombination in pristine and annealed bulk heterojunction solar cells. Appl. Phys. Lett. 2008, 93, No. 163303.

(12) Armin, A.; Velusamy, M.; Burn, P. L.; Meredith, P.; Pivrikas, A. Injected charge extraction by linearly increasing voltage for bimolecular recombination studies in organic solar cells. Appl. Phys. Lett. 2012, 101, No. 083306.

(13) Cowan, S. R.; Roy, A.; Heeger, A. J. Recombination in polymerfullerene bulk heterojunction solar cells. Phys. Rev. B 2010, 82, No. 245207.

(14) Koster, L. J. A.; Mihailetchi, V.; Blom, P. W. M. Bimolecular recombination in polymer/fullerene bulk heterojunction solar cells. Appl. Phys. Lett. 2006, 88, No. 052104.

(15) Juška, G.; Arlauskas, K.; Stuchlik, J.; Österbacka, R. NonLangevin bimolecular recombination in low-mobility materials. J. NonCryst. Solids 2006, 352, 1167-1171.

(16) Gasparini, N.; Salvador, M.; Heumueller, T.; Richter, M.; Classen, A.; Shrestha, S.; Matt, G. J.; Holliday, S.; Strohm, S.; Egelhaaf, H.; et al. Polymer: Nonfullerene Bulk Heterojunction Solar Cells with Exceptionally Low Recombination Rates. Adv. Energy Mater. 2017, 7, No. 1701561.

(17) Ju, H.; Knesting, K. M.; Zhang, W.; Pan, X.; Wang, C.; Yang, Y.; Ginger, D. S.; Zhu, J. Interplay between Interfacial Structures and Device Performance in Organic Solar Cells: A Case Study with the Low Work Function Metal, Calcium. ACS Appl. Mater. Interfaces 2016, $8,2125-2131$.

(18) Maurano, A.; Shuttle, C. G.; Hamilton, R.; Ballantyne, A. M.; Nelson, J.; Zhang, W.; Heeney, M.; Durrant, J. R. Transient optoelectronic analysis of charge carrier losses in a selenophene/ fullerene blend solar cell. J. Phys. Chem. C 2011, 115, 5947-5957.

(19) Foertig, A.; Wagenpfahl, A.; Gerbich, T.; Cheyns, D.; Dyakonov, V.; Deibel, C. Nongeminate recombination in planar and bulk heterojunction organic solar cells. Adv. Energy Mater. 2012, 2, 14831489 .

(20) Boix, P. P.; Ajuria, J.; Pacios, R.; Garcia-Belmonte, G. Carrier recombination losses in inverted polymer: Fullerene solar cells with $\mathrm{ZnO}$ hole-blocking layer from transient photovoltage and impedance spectroscopy techniques. J. Appl. Phys. 2011, 109, No. 074514.

(21) Sánchez-Díaz, A.; Burtone, L.; Riede, M.; Palomares, E. Measurements of efficiency losses in blend and bilayer-type zinc phthalocyanine/C60 high-vacuum-processed organic solar cells. J. Phys. Chem. C 2012, 116, 16384-16390.

(22) Ryan, J. W.; Palomares, E. Photo-Induced Charge Carrier Recombination Kinetics in Small Molecule Organic Solar Cells and the Influence of Film Nanomorphology. Adv. Energy Mater. 2017, 7, No. 1601509.

(23) Shuttle, C. G.; O’Regan, B.; Ballantyne, A. M.; Nelson, J.; Bradley, D. D.; Durrant, J. R. Bimolecular recombination losses in polythiophene: Fullerene solar cells. Phys. Rev. B 2008, 78, No. 113201

(24) Kniepert, J.; Schubert, M.; Blakesley, J. C.; Neher, D. Photogeneration and recombination in P3HT/PCBM solar cells probed by time-delayed collection field experiments. J. Phys. Chem. Lett. 2011, 2, 700-705.

(25) Kniepert, J.; Lange, I.; van der Kaap, N. J.; Koster, L. J. A.; Neher, D. A Conclusive View on Charge Generation, Recombination, and Extraction in As-Prepared and Annealed P3HT: PCBM Blends: Combined Experimental and Simulation Work. Adv. Energy Mater. 2014, 4, No. 1301401.

(26) Paulke, A.; Stranks, S. D.; Kniepert, J.; Kurpiers, J.; Wolff, C. M.; Schön, N.; Snaith, H. J.; Brenner, T. J.; Neher, D. Charge carrier recombination dynamics in perovskite and polymer solar cells. Appl. Phys. Lett. 2016, 108, No. 113505.

(27) Koster, L. J. A.; Kemerink, M.; Wienk, M. M.; Maturová, K.; Janssen, R. A. Quantifying bimolecular recombination losses in organic bulk heterojunction solar cells. Adv. Mater. 2011, 23, 1670-1674.

(28) Wheeler, S.; Deledalle, F.; Tokmoldin, N.; Kirchartz, T.; Nelson, J.; Durrant, J. R. Influence of surface recombination on charge-carrier kinetics in organic bulk heterojunction solar cells with nickel oxide interlayers. Phys. Rev. Appl. 2015, 4, No. 024020.

(29) Sandberg, O. J.; Sundqvist, A.; Nyman, M.; Österbacka, R. Relating Charge Transport, Contact Properties, and Recombination to Open-Circuit Voltage in Sandwich-Type Thin-Film Solar Cells. Phys. Rev. Appl. 2016, 5, No. 044005.

(30) Zonno, I.; Krogmeier, B.; Katte, V.; Lübke, D.; Martinez-Otero, A.; Kirchartz, T. Discriminating between surface and bulk recombination in organic solar cells by studying the thickness dependence of the open-circuit voltage. Appl. Phys. Lett. 2016, 109, No. 183301.

(31) Wagenpfahl, A.; Deibel, C.; Dyakonov, V. Organic solar cell efficiencies under the aspect of reduced surface recombination velocities. IEEE J. Sel. Top. Quantum Electron. 2010, 16, 1759-1763.

(32) Wehenkel, D. J.; Koster, L. J. A.; Wienk, M. M.; Janssen, R. A. Influence of injected charge carriers on photocurrents in polymer solar cells. Phys. Rev. B 2012, 85, No. 125203.

(33) Cowan, S. R.; Li, J. V.; Olson, D. C.; Ratcliff, E. L. ContactInduced Mechanisms in Organic Photovoltaics: A Steady-State and Transient Study. Adv. Energy Mater. 2015, 5, No. 1400549.

(34) Reinhardt, J.; Grein, M.; Bühler, C.; Schubert, M.; Würfel, U. Identifying the impact of surface recombination at electrodes in organic solar cells by means of electroluminescence and modeling. Adv. Energy Mater. 2014, 4, No. 1400081.

(35) Yoon, Y.; Kim, H. J.; Cho, C.; Kim, S.; Son, H. J.; Ko, M.; Kim, H.; Lee, D.; Kim, J. Y.; Lee, W.; et al. Carrier lifetime extension via the incorporation of robust hole/electron blocking layers in bulk heterojunction polymer solar cells. ACS Appl. Mater. Interfaces 2014, $6,333-339$.

(36) Zhang, X.; Li, W.; Yao, J.; Zhan, C. High-efficiency nonfullerene polymer solar cell enabling by integration of film-morphology optimization, donor selection, and interfacial engineering. ACS Appl. Mater. Interfaces 2016, 8, 15415-15421.

(37) Singh, A.; Dey, A.; Das, D.; Iyer, P. K. Effect of Dual Cathode Buffer Layer on the Charge Carrier Dynamics of rrP3HT: PCBM Based Bulk Heterojunction Solar Cell. ACS Appl. Mater. Interfaces 2016, 8, 10904-10910.

(38) Hains, A. W.; Marks, T. J. High-efficiency hole extraction/ electron-blocking layer to replace poly (3, 4-ethylenedioxythiophene): poly (styrene sulfonate) in bulk-heterojunction polymer solar cells. Appl. Phys. Lett. 2008, 92, No. 023504.

(39) Venkatesan, S.; Ngo, E.; Khatiwada, D.; Zhang, C.; Qiao, Q. Enhanced lifetime of polymer solar cells by surface passivation of metal oxide buffer layers. ACS Appl. Mater. Interfaces 2015, 7, 16093-16100.

(40) Yeo, J.-S.; Yun, J.; Kang, M.; Khim, D.; Lee, S.; Kim, S.; Na, S.; Kim, D. An approach for an advanced anode interfacial layer with electron-blocking ability to achieve high-efficiency organic photovoltaics. ACS Appl. Mater. Interfaces 2014, 6, 19613-19620.

(41) Petersen, A.; Kirchartz, T.; Wagner, T. A. Charge extraction and photocurrent in organic bulk heterojunction solar cells. Phys. Rev. B 2012, 85, No. 045208 .

(42) Bartesaghi, D.; del Carmen Pérez, I.; Kniepert, J.; Roland, S.; Turbiez, M.; Neher, D.; Koster, L. J. A. Competition between recombination and extraction of free charges determines the fill factor of organic solar cells. Nat. Commun. 2015, 6, No. 7083.

(43) Le Corre, V. M.; Chatri, A. R.; Doumon, N. Y.; Koster, L. J. A. Charge Carrier Extraction in Organic Solar Cells Governed by SteadyState Mobilities. Adv. Energy Mater. 2017, 7, No. 1701138.

(44) Selberherr, S. Analysis and Simulation of Semiconductor Devices; Springer-Verlag: Wien, 1984

(45) Lakhwani, G.; Rao, A.; Friend, R. H. Bimolecular recombination in organic photovoltaics. Annu. Rev. Phys. Chem. 2014, 65, 557-581.

(46) Foertig, A.; Kniepert, J.; Gluecker, M.; Brenner, T.; Dyakonov, V.; Neher, D.; Deibel, C. Nongeminate and geminate recombination in PTB7: PCBM solar cells. Adv. Funct. Mater. 2014, 24, 1306-1311. 